\title{
Infectious uveitis in Virginia
}

\author{
Stephanie B Engelhard' \\ Zeina Haddad' \\ Asima Bajwa' \\ James Patrie ${ }^{2}$ \\ Wenjun $\mathrm{Xin}^{2}$ \\ Ashvini K Reddy' \\ 'Department of Ophthalmology, \\ ${ }^{2}$ Department of Public Health \\ Sciences, University of Virginia, \\ Charlottesville, VA, USA
}

This article was published in the following Dove Press journal:

Clinical Ophthalmology

28 August 2015

Number of times this article has been viewed

Purpose: To report the causes, clinical features, and outcomes of infectious uveitis in patients managed in a mid-Atlantic tertiary care center.

Methods: Retrospective, observational study of infectious uveitis patients seen at the University of Virginia from 1984 to 2014.

Results: Seventy-seven of 491 patients (15.7\%) were diagnosed with infectious uveitis (mean age 58 years, $71.4 \%$ female, $76.6 \%$ Caucasian). The mean follow-up was 5 years. Anterior uveitis was the most common anatomic classification ( 39 patients, $50.6 \%$ ) followed by panuveitis ( 20 patients, $26.0 \%$ ) and posterior uveitis (18 patients, $23.4 \%$ ). The most common infectious etiology was herpetic anterior uveitis ( 37 patients, $48.1 \%$ ) followed by toxoplasma uveitis (14 patients, $18.2 \%$ ). The most prevalent viral pathogen was varicella-zoster virus (21 patients, 27.3\%) followed by herpes simplex virus (20 patients, $26.0 \%$ ). Acute retinal necrosis (ARN) was diagnosed in 14 patients (18.2\%). Aqueous humor yielded an etiologic diagnosis in seven $(50 \%)$ of ARN patients, four of whom tested positive for cytomegalovirus and three for varicella-zoster virus. On presentation, 43 patients (55.8\%) had a visual acuity (VA) better than 20/40 and $17(22.1 \%)$ had a VA worse than 20/200. VA at the final follow-up was better than 20/40 in 39 patients $(50.6 \%)$ and worse than $20 / 200$ in 22 patients $(28.6 \%)$. In all, $16(20.8 \%)$ and $10(13.0 \%)$ patients required cataract and vitrectomy surgery, respectively. A total of 14 patients $(18.2 \%)$ were on glaucoma topical treatment and four (5.2\%) required glaucoma surgery.

Conclusion: The most common type of infectious uveitis seen over the study period was herpetic anterior uveitis secondary to varicella-zoster virus or herpes simplex virus, found to be most prevalent in patients over 60 years of age. This finding is comparable to other American epidemiologic studies. Ocular toxoplasmosis and ARN were also common causes of infectious uveitis. In all, $50.6 \%$ of patients had a VA better than 20/40 at the final follow-up visit, indicating the importance of prompt referral and appropriate treatment.

Keywords: uveitis, infectious, chorioretinitis, endophthalmitis, toxoplasma

\section{Introduction}

Uveitis includes a wide range of inflammatory eye diseases representing a diverse set of both infectious and non-infectious etiologies. Globally, uveitis accounts for up to $25 \%$ of blindness. ${ }^{1}$ Although this percentage is lower in developed countries than in developing countries, uveitis still accounts for $10 \%-15 \%$ of blindness in the United States, representing a devastating loss of quality of life and productivity to those affected. ${ }^{1-5}$

Infectious uveitis includes bacterial, viral, fungal, and parasitic etiologies. The prevalence of specific infectious etiologies varies historically and with geographic location. ${ }^{6}$ In the West, infectious uveitis is often associated with systemic diseases, including diseases caused by Toxoplasma gondii, varicella-zoster virus (VZV), and herpes simplex virus (HSV) and, less often, syphilis and tuberculosis (TB). ${ }^{6,7}$ Previous studies from around the world have focused primarily on describing the incidence and prevalence of all uveitis etiologies at different referral centers. Studies of infectious
Correspondence: Ashvini K Reddy Department of Ophthalmology, University of Virginia, I 300 Jefferson Park Avenue, Charlottesville, VA 22908, USA

Tel +l 4342435890

Fax +I 4349245180

Email akr5u@hscmail.mcc.virginia.edu 
uveitis have either focused on a specific infectious etiology or on a specific demographic variable..$^{7-11}$ This retrospective, observational study was performed in order to report all infectious uveitis cases at the University of Virginia, a mid-Atlantic US tertiary referral center, over a 30-year period and to analyze the causes, clinical features, and outcomes in this population.

\section{Methods}

This was a retrospective, observational study of all patients with infectious uveitis seen in the Department of Ophthalmology at the University of Virginia. The study was approved by the Institutional Review Board and was conducted in accordance with the principles of the Declaration of Helsinki. A database of all patients with a diagnosis of uveitis is maintained in the Department of Ophthalmology at the University of Virginia and includes 491 patients. The database was reviewed to identify patients diagnosed with infectious uveitis over a 30-year period from 1984 through 2014. Seventy-seven infectious uveitis patients were identified from the database. No minimum follow-up period was required for inclusion, and all data in the database were collected from patient charts completed at the time of patient visits. Time points for data collection in the database included patients' initial visit to the clinic (baseline) and the final follow-up visit. Disease classification was recorded in accordance with the Standardization of Uveitis Nomenclature (SUN) Working Group criteria. ${ }^{12}$ The database was reviewed for patient demographic information, including date of birth, sex, race/ethnicity, age at presentation, and duration of follow-up. Clinical information including final diagnosis with infectious etiology; location, laterality, and chronicity of uveitis; best-corrected visual acuity (VA); intraocular pressure; laboratory findings; relevant systemic and associated diagnoses; treatment modalities; and complications were also reviewed. Good visual outcome was defined as $\mathrm{VA} \geq 20 / 40$, moderate visual impairment was defined as a VA between 20/50 and 20/200, and severe visual impairment was defined as VA worse than 20/200. ${ }^{12}$ Uveitis etiologies were confirmed by imaging with optical coherence tomography, fluorescein angiography, and automated perimetry and by further ancillary testing, including serology, radiology, microbiology, and biopsy when appropriate. Intraocular infection was confirmed with fluid sampling or biopsy for microscopy and cytology and culture or polymerase chain reaction, when appropriate. Management including the use of local and systemic steroids, antihypertensive drops, intravitreal injections, subtenon steroid injections, antimetabolites, anti-tumor necrosis factor agents, cataract surgery, pars plana vitrectomy, and glaucoma management (medical and surgical intervention) was recorded. Owing to the small nature of the data set, descriptive statistical analysis was performed. Randomization tests were used to determine significance where appropriate.

\section{Results}

A review of all 491 patients diagnosed with uveitis at the University of Virginia between 1984 and 2004 was conducted. Seventy-seven patients (92 eyes) diagnosed with infectious uveitis were identified from the 491 patients (15.7\%) included in the study.

Of the 77 patients, $55(71.4 \%)$ were female and only 22 $(28.6 \%)$ were male. Fifty-nine patients $(76.6 \%)$ were Caucasian, nine (11.7\%) were African American, two (2.6\%) were Hispanic, and seven $(9.1 \%)$ were of another race. The mean age at uveitis diagnosis was 58.2 years (standard deviation, 20.3). The mean duration of follow-up time was 5 years. The total follow-up time ranged from 4 days to 30 years. Sixtytwo patients $(80.5 \%)$ had unilateral disease and 15 patients $(19.5 \%)$ had bilateral disease. Bilateral disease was seen in two of 14 patients $(14.3 \%)$ with toxoplasma, one of 19 patients $(5.3 \%)$ with herpetic anterior uveitis secondary to $\mathrm{HSV}$, five of 12 patients $(41.7 \%)$ with herpetic anterior uveitis secondary to herpes zoster ophthalmicus (HZO), two of two patients $(100 \%)$ with acute retinal necrosis (ARN) secondary to cytomegalovirus (CMV), three of four patients $(75 \%)$ with ARN, and two of three patients $(67 \%)$ with CMV retinitis.

Forty-five eyes of 39 patients were diagnosed with anterior infectious uveitis, which was the most common anatomical location. Twenty-three eyes of 18 patients were diagnosed with infectious posterior uveitis, 24 eyes of 20 patients were diagnosed with infectious panuveitis, and there were no diagnoses of intermediate uveitis. The most common uveitis diagnosis in our population was herpetic anterior uveitis $(n=37,48.1 \%)$. Of those 37 patients, the etiologic agent was HSV in 20 patients $(26.0 \%)$ and $\mathrm{HZO}$ in 17 patients $(22.1 \%)$. After herpetic anterior uveitis, the next most common etiology was toxoplasma uveitis $(n=14$, $18.2 \%$ ). ARN was diagnosed in 14 patients (18.2\%). Aqueous humor was analyzed in all 14 ARN patients and was positive in seven patients (50\%). Four of the 14 ARN patients tested positive for CMV and three were positive for VZV. Only five patients $(6.5 \%)$ had classic CMV retinitis, and three patients $(3.9 \%)$ had fungal endophthalmitis. Other diagnoses found in one patient each included TB uveitis, syphilitic 
chorioretinitis, fungal sclerokeratitis following penetrating keratoplasty, and herpetic panuveitis secondary to HZO. There was a marked female predominance $(75.7 \%)$ seen in patients with herpetic anterior uveitis. Only four of the 77 patients $(5.2 \%)$ were 19 years old or younger at the time of diagnosis. The majority of the patients ( 38 of 77 patients [49.4\%]) were at least 60 years old at diagnosis. In this series, advanced age was found to be a significant indicator for higher prevalence of uveitis secondary to toxoplasma infection $(P=0.014)$, herpetic anterior uveitis secondary to HZO ( $P=0.017)$, and ARN secondary to VZV $(P=0.020)$. Table 1 summarizes the distribution of uveitis diagnoses by age.

The most common etiologies of anterior uveitis were herpetic anterior uveitis secondary to HSV (20 of 39 patients [51.3\%]) and herpetic anterior uveitis secondary to HZO (17 of 39 patients [43.6\%]). Other anterior uveitis diagnoses included one case each of fungal sclerokeratitis secondary to penetrating keratoplasty and ARN secondary to CMV. Eleven of 18 cases $(61.1 \%)$ of posterior uveitis were due to toxoplasma infection. Other posterior uveitis diagnoses included ARN secondary to CMV (three of 18 patients [16.7\%]), CMV retinitis (three of 18 patients [16.7\%]), and syphilitic chorioretinitis (one of 18 patients [5.6\%]). The most common etiology of panuveitis was ARN (seven of 20 patients [35.0\%]). Other panuveitis diagnoses included toxoplasma uveitis (15.0\%), fungal endophthalmitis (15.0\%), TB uveitis (5.0\%), ARN secondary to VZV (15.0\%), herpetic panuveitis secondary to $\mathrm{HZO}(5.0 \%)$, and CMV retinitis $(10.0 \%)$.

Ophthalmic management and interventions were documented. During the follow-up period, 49 of the 77 patients $(63.6 \%)$ received local steroids and $21(27.3 \%)$ received systemic steroids. Antimetabolites were prescribed to five $(6.5 \%)$ of the patients, and two $(2.6 \%)$ of the patients were prescribed an antitumor necrosis factor agent. One (1.3\%) was given subtenon injections and six (7.8\%) were given intravitreal injections. Sixteen patients (20.8\%) underwent cataract surgery, and pars plana vitrectomy was performed in ten patients (13.0\%). Fourteen patients $(18.2 \%)$ who developed glaucoma were managed medically, and four patients (5.2\%) underwent glaucoma surgery. On presentation, 43 of 77 patients $(55.8 \%)$ had a visual acuity of $20 / 40$ or better, 17 patients (22.1\%) had a visual acuity between 20/50 and $20 / 200$, and 17 patients (22.1\%) had a visual acuity of worse than $20 / 200$. At the final follow-up, 39 of the 77 patients $(50.6 \%)$ had a good visual outcome of $20 / 40$ or better. Sixteen patients $(20.8 \%)$ had moderate visual impairment between 20/50 and 20/200 at the final follow-up visit, and 22 patients $(28.6 \%)$ experienced severe visual impairment of $<20 / 200$ at the time of final visit. In this series, patients with a diagnosis of uveitis secondary to toxoplasma infection were significantly more likely to have a good visual outcome of $20 / 40$ or better than a poor outcome $(P=0.006)$, with ten of 14 patients (71.4\%) having a final visual acuity of $\geq 20 / 40$. Infections with herpetic anterior uveitis secondary to $\mathrm{HZO}$ were also significantly more likely to result in good visual outcomes of $20 / 40$ or better than poor outcomes $(P<0.001)$, with 15 of 17 patients $(88.2 \%)$ having a visual acuity of $\geq 20 / 40$ at the final follow-up. The poorest visual outcomes were observed in fungal endophthalmitis, with all three fungal endophthalmitis patients having severe visual impairment of 20/200 or worse at the final follow-up $(P=0.540)$. Visual acuities by diagnosis at the final follow-up visit are found in Table 2 .

Table I Infectious uveitis diagnosis distribution by age (years)

\begin{tabular}{|c|c|c|c|c|c|c|}
\hline \multirow[t]{2}{*}{ Diagnosis } & \multicolumn{4}{|c|}{ Age group (years) } & \multirow[t]{2}{*}{ Total } & \multirow[t]{2}{*}{$P$-value } \\
\hline & $0-19$ & $20-39$ & $40-59$ & $\geq 60$ & & \\
\hline Toxoplasma & 0 & 2 & 4 & 8 & 14 & $0.014^{*}$ \\
\hline Fungal endophthalmitis & 0 & 1 & 0 & 2 & 3 & 0.564 \\
\hline Tuberculosis & 0 & 0 & 0 & I & I & 0.317 \\
\hline Syphilis & 0 & 0 & 0 & I & I & 0.317 \\
\hline Fungal sclerokeratitis s/p PK & 0 & 0 & 0 & I & $\mathrm{I}$ & 0.317 \\
\hline Herpetic anterior uveitis (HSV) & 2 & 5 & 7 & 6 & 20 & 0.796 \\
\hline Herpetic anterior uveitis ( $\mathrm{HZO})$ & 0 & 2 & 6 & 9 & 17 & $0.017^{*}$ \\
\hline Acute retinal necrosis (CMV) & 0 & 0 & 2 & 2 & 4 & 0.564 \\
\hline Acute retinal necrosis** & I & 1 & I & 4 & 7 & 0.127 \\
\hline Acute retinal necrosis (VZV) & 0 & 0 & 0 & 3 & 3 & $0.020 *$ \\
\hline Herpetic panuveitis (HZO) & 0 & 0 & 0 & I & I & 0.317 \\
\hline CMV retinitis & 1 & 2 & 2 & 0 & 5 & 0.437 \\
\hline Total & 4 & 13 & 22 & 38 & 77 & \\
\hline
\end{tabular}

Notes: *Statistically significant at $5 \%$ level. **No virus isolated.

Abbreviations: PK, penetrating keratoplasty; HSV, herpes simplex virus; HZO, herpes zoster ophthalmicus; CMV, cytomegalovirus; VZV, varicella-zoster virus. 
Table 2 Visual acuity at the final follow-up by diagnosis

\begin{tabular}{|c|c|c|c|c|c|}
\hline Diagnosis & $\begin{array}{l}\geq 20 / 40 \\
\text { No (\%) }\end{array}$ & $\begin{array}{l}20 / 50-20 / 200 \\
\text { No (\%) }\end{array}$ & $\begin{array}{l}<20 / 200 \\
\text { No (\%) }\end{array}$ & $\begin{array}{l}\text { Total no } \\
\text { of patients }\end{array}$ & P-value \\
\hline Toxoplasma & $10(7 \mathrm{I} .4)$ & $2(14.3)$ & $2(14.3)$ & 14 & $0.006 *$ \\
\hline Fungal endophthalmitis & $0(0)$ & $0(0)$ & $3(100)$ & 3 & 0.540 \\
\hline Tuberculosis & I (I00) & $0(0)$ & $0(0)$ & 1 & 0.724 \\
\hline Syphilis & I (100) & $0(0)$ & $0(0)$ & $\mathrm{I}$ & 0.724 \\
\hline Fungal sclerokeratitis s/p PK & $0(0)$ & $0(0)$ & I (I00) & 1 & 0.479 \\
\hline Herpetic anterior uveitis (HSV) & $7(35.0)$ & $9(45.0)$ & $4(20.0)$ & 20 & 0.874 \\
\hline Herpetic anterior uveitis ( $\mathrm{HZO}$ ) & $15(88.2)$ & I (5.9) & I (5.9) & 17 & $<0.00 I^{*}$ \\
\hline Acute retinal necrosis (CMV) & I (25.0) & I (25.0) & $2(50.0)$ & 4 & 0.504 \\
\hline Acute retinal necrosis** & I (I4.3) & $2(28.6)$ & $4(57.1)$ & 7 & 0.504 \\
\hline Acute retinal necrosis (VZV) & I (33.3) & $0(0)$ & $2(66.7)$ & 3 & 0.724 \\
\hline Herpetic panuveitis (HZO) & $0(0)$ & $0(0)$ & I (100) & 1 & 0.480 \\
\hline CMV retinitis & $2(40.0)$ & I (20.0) & $2(40.0)$ & 5 & 0.752 \\
\hline Total & $39(50.6)$ & $16(20.8)$ & $22(28.6)$ & 77 & \\
\hline
\end{tabular}

Notes: *Statistically significant at $5 \%$ level. **No virus isolated.

Abbreviations: PK, penetrating keratoplasty; HSV, herpes simplex virus; HZO, herpes zoster ophthalmicus; CMV, cytomegalovirus; VZV, varicella-zoster virus.

\section{Discussion}

This retrospective study seeks to report the demographics, etiologies, treatments, and outcomes of a population of 77 infectious uveitis patients at a tertiary referral center in Virginia, USA. Over a 30-year period, 491 patients with uveitis were seen at the ophthalmology clinic at the University of Virginia. Of those 491 patients, 77 (15.7\%) were diagnosed with infectious uveitis. This falls on the low end of the range reported in the literature, largely because this study was conducted in a developed country. One study found infectious etiologies in $23.1 \%$ of uveitis cases in an Iranian tertiary referral center. ${ }^{13}$ Similarly, a Lebanese study found infectious etiologies in $24 \%$ of patients. ${ }^{1}$ The percentage of infectious uveitis cases reported in developed countries is lower and more in line with this series. One Boston study reported infectious etiologies in $16.9 \%$ of uveitis cases. ${ }^{6}$ A study of pediatric infectious uveitis in the Netherlands reported a prevalence of infectious etiologies of $17 \%$. $^{7}$ The female to male ratio in our study was $2.5: 1$, which is considerably higher than that reported in other studies. This is largely due to the difference in prevalence of anterior uveitis secondary to HSV and HZO by sex observed in this series. While only nine male patients were diagnosed with either HSV or HZO anterior uveitis, 28 female patients received these diagnoses, meaning that $75.7 \%$ of HSV and HZO patients were female. In two Saudi studies, males with herpetic anterior uveitis significantly outnumbered females with this diagnosis. ${ }^{14,15}$ In a Boston study, prevalence of HSV and $\mathrm{HZO}$ anterior uveitis was roughly equal between males and females. ${ }^{6}$ These sex differences likely result from referral bias across different geographic locations.
The most prevalent viral pathogen in our population was $\operatorname{VZV}(n=21,27.3 \%)$ followed by HSV ( $n=20,26.0 \%)$. In one Boston study, systemic infection with HSV and VZV was found in $5.0 \%$ of uveitis patients. ${ }^{6}$ A Lebanese study reported herpetic etiologies in $6.2 \%$ of patients and a San Francisco study reported herpetic etiologies in $8.5 \%$. ${ }^{1,2}$ In our population, the 41 patients found to have systemic HSV or VZV infection represent $8.4 \%$ of the 491 uveitis patients seen at our site over the last 30 years, which is consistent with previous studies. Of the 41 patients with systemic HSV or VZV infection, 20 had HSV anterior uveitis, 17 had HZO anterior uveitis, three had ARN secondary to VZV, and one had HZO panuveitis. Together, herpetic anterior uveitis secondary to HSV and HZO accounted for $48.1 \%$ of all infectious uveitis cases in our population and was especially prevalent in patients over the age of 60 .

After herpetic uveitis, the next most common etiology of infectious uveitis in our population was secondary to toxoplasma infection. In our population, $18.2 \%$ of all infectious uveitis cases were attributable to toxoplasma infection, representing $2.9 \%$ of all uveitis cases over the past 30 years. This is consistent with reports from other American centers, which reported toxoplasma uveitis in $4.8 \%$ of patients in a Boston study and $4 \%$ of patients in a San Francisco study., Prevalence of toxoplasma uveitis was often higher in international centers, representing $28 \%$ of all uveitis cases in a Saudi study and 19\% in an Iranian study, which reflects differences in toxoplasma infection rates by geographic location. ${ }^{13,14}$

TB uveitis accounted for only $1.3 \%$ of infectious uveitis and only $0.2 \%$ of all uveitis cases in our population. This is consistent with reports from other US sites, which report TB 
uveitis prevalences of $0.4 \%$ and $0.6 \%,,^{2,6}$ and considerably lower than international sites, which report prevalences of 1.62\% (India), 3.4\% (Japan), 5.7\% (Lebanon), and 7\% and $17.8 \%$ (Saudi Arabia). ${ }^{1,14-17}$ Similarly, syphilitic chorioretinitis was found in only one patient in our population, representing $1.3 \%$ of infectious uveitis and only $0.2 \%$ of all uveitis cases. This is similar to that reported in the literature: $0.3 \%$ (India), $0.5 \%$ (Lebanon), and $1.7 \%$ (Boston). ${ }^{1,6,16}$ Overall, $50.6 \%$ of patients had a good visual outcome of 20/40 or better. Moderate visual impairment between 20/50 and $20 / 200$ was observed in $20.8 \%$ of patients, and severe visual impairment was observed in $28.6 \%$ of patients. Patients with toxoplasma uveitis $(P=0.006)$ and herpetic anterior uveitis secondary to HZO $(P<0.001)$ experienced the best visual outcomes and were significantly more likely to have a good visual outcome than a poor outcome. Ten of the 14 toxoplasma uveitis patients $(71.4 \%)$ had a visual acuity of $20 / 40$ or better at the final follow-up, while only two toxoplasma patients $(14.3 \%)$ had moderate visual impairment and two patients $(14.3 \%)$ had severe visual impairment at the final visit. These results are consistent with previous studies. In one Saudi study, $68 \%$ of patients with toxoplasma uveitis had good visual outcomes, while $21 \%$ experienced moderate visual impairment and only $11 \%$ experienced severe visual impairment. ${ }^{14}$ Similar results were found in a Netherlands study, which reported good visual outcomes in $65 \%$ of toxoplasma patients, moderate visual impairment in $20 \%$ of patients, and severe visual impairment in only $15 \%$ of patients. $^{3}$

In our population, patients with herpetic anterior uveitis secondary to $\mathrm{HZO}$ also had good visual outcomes with 15 of 17 patients $(88.2 \%)$ having visual acuity of $20 / 40$ or better at the final follow-up. Patients with herpetic anterior uveitis secondary to HSV also had good visual outcomes overall. Most patients with HSV anterior uveitis had visual outcomes of $20 / 40$ or better $(35.0 \%)$ or between $20 / 50$ and $20 / 200$ (45.0\%). Visual results for ARN were mixed. Although numbers of patients with ARN were too small to be statistically significant, it seems that VZV- and CMV-negative ARN patients had worse outcomes than ARN patients who were positive for VZV or CMV. Overall, visual outcomes in this study reveal the importance of proper diagnosis and treatment in order to increase the likelihood of having good visual outcomes.

The major weaknesses of this study include the limited sample size as well as the retrospective nature of the study, both of which make the study susceptible to referral, treatment, and data collection biases. Because there was not a standardized follow-up period required for inclusion in the study, it is possible that the visual outcomes represent only patients with more severe disease or those with longer follow-up periods. Because the data were collected over the past 30 years and because management protocols and diagnostic and treatment modalities have changed over time, it is possible that visual outcomes, complications, and treatments reported in this study may have evolved. Owing to the above limitations, statistical analysis was largely descriptive in nature. Although the limitations described here prevent broad generalization of our results, to our knowledge, this report is one of the only reports in the literature that analyses all infectious uveitis cases over a significant period of time.

In conclusion, our study demonstrates the complexity of diagnosing and managing infectious uveitis due to the great number of possible etiologies. As we have demonstrated, the expected outcomes of infectious uveitis depend on numerous factors, including age, sex, and specific infectious etiology. Because the visual outcomes vary considerably with these demographic and etiologic variables, prompt referral to a uveitis specialist is important to establish diagnosis and to begin appropriate management in order to prevent long-term ocular damage and vision loss. In our population, $50.6 \%$ of patients had a VA better than 20/40 at the final follow-up, again highlighting the importance of prompt referral and appropriate treatment.

\section{Meeting presentation}

A portion of this study has been accepted for poster presentation at the Association for Research in Vision and Ophthalmology (ARVO), June 2015.

\section{Acknowledgment}

The authors received no financial support for this study.

\section{Disclosure}

The authors report no conflicts of interest in this work.

\section{References}

1. Abdulaal M, Antonios R, Barikian A, Jaroudi M, Hamam RN. Etiology and clinical features of ocular inflammatory diseases in a tertiary center in Lebanon. Ocul Immunol Inflamm. Epub 2014 Jun 9.

2. Acharya NR, Tham VM, Esterberg E, et al. Incidence and prevalence of uveitis: results from the Pacific Ocular Inflammation Study. JAMA Ophthalmol. 2013;131(11):1405-1412.

3. Rothova A, Suttorp-Van Schulten MS, Treffers WF, Kijlstra A. Causes and frequency of blindness in patients with intraocular inflammatory disease. Br J Ophthalmol. 1996;80(4):332-336.

4. Suttorp-Schulten MS, Rothova A. The possible impact of uveitis in blindness: a literature survey. Br J Ophthalmol. 1996;80(9):844-848. 
5. Suhler EB, Lloyd MJ, Choi D, Rosenbaum JT, Austin DF. Incidence and prevalence of uveitis in Veterans Affairs Medical Centers of the Pacific Northwest. Am J Ophthalmol. 2008;146(6):890-896.

6. Rodriguez A, Calonge M, Pedroza-Seres M, et al. Referral patterns of uveitis in a tertiary eye care center. Arch Ophthalmol. 1996; 114(5):593-599.

7. Hettinga YM, de Groot-Mijnes JD, Rothova A, de Boer JH. Infectious involvement in a tertiary center pediatric uveitis cohort. Br J Ophthalmol. 2015;99(1):103-107.

8. Reich M, Ruppenstein M, Becker MD, Mackensen F. Time patterns of recurrences and factors predisposing for a higher risk of recurrence of ocular toxoplasmosis. Retina. 2015;35(4):809-819.

9. Bosch-Driessen LE, Berendschot TT, Ongkosuwito JV, Rothova A. Ocular toxoplasmosis: clinical features and prognosis of 154 patients. Ophthalmology. 2002;109(5):869-878.

10. Wensing B, Relvas LM, Caspers LE, et al. Comparison of rubella virusand herpes virus-associated anterior uveitis: clinical manifestations and visual prognosis. Ophthalmology. 2011;118(10):1905-1910.

11. Miserocchi E, Waheed NK, Dios E, et al. Visual outcome in herpes simplex virus and varicella zoster virus uveitis: a clinical evaluation and comparison. Ophthalmology. 2002;109(8):1532-1537.
12. Standardization of Uveitis Nomenclature (SUN) Working Group. Standardization of uveitis nomenclature for reporting clinical data. Results of the first international workshop. Am J Ophthalmol. 2005;140(3):509-516.

13. Rahimi M, Mirmansouri G. Patterns of uveitis at a tertiary referral center in southern Iran. J Ophthalmic Vis Res. 2014;9(1):54-59.

14. Hamade IH, Elkum N, Tabbara KF. Causes of uveitis at a referral center in Saudi Arabia. Ocul Immunol Inflamm. 2009;17(1):11-16.

15. Al Dhahri H, Al Rubaie K, Hemachandran S, et al. Patterns of uveitis in a university-based tertiary referral center in Riyadh, Saudi Arabia. Ocul Immunol Inflamm. Epub 2014 Jul 24.

16. Das D, Bhattacharjee H, Bhattacharyya PK, et al. Pattern of uveitis in North East India: a tertiary eye care center study. Indian J Ophthalmol. 2009;57(2):144.

17. Wakabayashi T, Morimura Y, Miyamoto Y, Okada AA. Changing patterns of intraocular inflammatory disease in Japan. Ocul Immunol Inflamm. 2003;11(4):277-286.

\section{Clinical Ophthalmology}

\section{Publish your work in this journal}

Clinical Ophthalmology is an international, peer-reviewed journal covering all subspecialties within ophthalmology. Key topics include: Optometry; Visual science; Pharmacology and drug therapy in eye diseases; Basic Sciences; Primary and Secondary eye care; Patient Safety and Quality of Care Improvements. This journal is indexed on

\section{Dovepress}

PubMed Central and CAS, and is the official journal of The Society of Clinical Ophthalmology (SCO). The manuscript management system is completely online and includes a very quick and fair peer-review system, which is all easy to use. Visit http://www.dovepress.com/ testimonials.php to read real quotes from published authors. 